\title{
Extensively drug-resistant tuberculosis in the UK: 1995 to 2007
}

\author{
I Abubakar, ${ }^{1}$ J Moore, ${ }^{1}$ F Drobniewski, ${ }^{1}$ M Kruijshaar, ${ }^{1}$ T Brown, ${ }^{1}$ M Yates, ${ }^{1}$ \\ C Anderson, ${ }^{1}$ E G Smith, ${ }^{2}$ J Magee, ${ }^{3}$ M Lipman, ${ }^{4}$ J McMenamin, ${ }^{5}$ M Ruddy, ${ }^{6}$ \\ J M Watson ${ }^{1}$
}

\begin{abstract}
${ }^{1}$ Respiratory and Systemic Infections Department, Centre for Infections, Health Protection Agency, London, UK; ${ }^{2}$ Health Protection Agency Regional Centre for Mycobacteriology, Heart of England NHS Foundation Trust, Bordesley Green East, Birmingham, UK ;

${ }^{3}$ Health Protection Agency Regional Centre for

Mycobacteriology, General Hospital, Westgate Road,

Newcastle upon Tyne, UK;

${ }^{4}$ Royal Free Hospital NHS Trust,

Pond Street London, UK;

${ }^{5}$ Health Protection Scotland, Glasgow, UK; ${ }^{6}$ Wales Centre for Mycobacteriology, Llandough Hospital, Penlan Road, Penarth, UK
\end{abstract}

Correspondence to: Dr I Abubakar, Tuberculosis Section, Respiratory and Systemic Infections Department, Centre for Infections, Health

Protection Agency, London NW9 5E0; Ibrahim.abubakar@ hpa.org.uk

Received 26 September 2008 Accepted 7 February 2009

Published online first

23 March 2009

\begin{abstract}
Background: The emergence of multidrug-resistant tuberculosis (MDRTB) and extensively drug-resistant tuberculosis (XDRTB) is a threat to global tuberculosis control. Limited information is, however, available on the outcome of XDRTB cases. This study describes the susceptibility to second- and third-line antituberculosis drugs among MDRTB cases and treatment outcome of identified XDRTB cases.
\end{abstract}

Method: The results of second-line antituberculosis drug susceptibility tests in the UK between January 1995 and December 2007 were retrospectively reviewed and clinicians contacted for treatment outcome of XDRTB cases. Participants included all 678 patients with cultureconfirmed MDRTB in the UK. The main outcome measures were the proportion of isolates resistant to second-line antituberculosis drugs and treatment outcome for XDRTB cases.

Results: Among MDRTB isolates, levels of resistance to amikacin, capreomycin, ciprofloxacin, cycloserine, ethionamide and $p$-aminosalicylic acid (PAS) were 5.5, 3.4, 5.6, 5.1, 14.0 and $16.7 \%$, respectively. Six XDRTB cases $(0.9 \%$ of MDR cases) were identified during this period. Two further cases of XDRTB were reported in 2008. Five individuals with XDRTB died of tuberculosis within 3 years of diagnosis and three are still on treatment.

Conclusion: Levels of MDRTB remain low, and those of XDRTB very low, in this high income country. The case fatality ratio among XDRTB cases was high despite low levels of HIV co-infection.

The emergence of multidrug-resistant tuberculosis (MDRTB) and extensively drug-resistant tuberculosis (XDRTB) is a threat to global tuberculosis control. These forms of tuberculosis are difficult and expensive to treat and often associated with a poor clinical outcome. ${ }^{12}$ In the case of MDRTB, this relates to the loss of the highly potent antituberculosis drugs isoniazid, the drug with the strongest early bactericidal action against Mycobacterium tuberculosis complex, and rifampicin, which acts against bacilli that are no longer in the active phase of replication. Treatment options are fairly limited and associated with a variety of side effects. ${ }^{3}$ Although survival is improved by the administration of drugs to which the bacteria are susceptible, ${ }^{14}$ the development of resistance to second- and third-line drugs (especially fluoroquinolones and injectable agents) among cases with MDRTB further worsens the prognosis of these patients. ${ }^{2}$

The World Health Organization describes increasing levels of MDRTB globally. ${ }^{5}$ In the UK, the incidence of MDRTB has risen, although the magnitude of the increase is small. ${ }^{6}$ Here we describe the susceptibility to second- and third-line antituberculosis drugs among MDRTB cases in the UK between January 1995 and December 2007 and report the number, characteristics and treatment outcome of identified XDRTB cases.

\section{METHODS}

\section{Study population}

The results of drug susceptibility testing of all $M$ tuberculosis complex isolates undertaken between 1995 and 2007 have been collated through the UK Mycobacterial Surveillance Network (MycobNet) collaboration. Results of drug susceptibility to the six main classes of drugs used to treat MDRTB (fluoroquinolones, serine analogues, aminoglycosides, capreomycin, $p$-aminosalicylic acid (PAS) and the thionamides) and other agents (clarithromycin, azithromycin, rifabutin, streptomycin and clofazimine) were analysed. Duplicate results from isolates were manually removed by using patient names, date of birth and address where available. It is, however, possible that a small number of duplicate records remain due to the limited amount of demographic information in the MycobNet database.

\section{Definitions}

MDRTB was defined as resistance to at least isoniazid and rifampicin, and XDRTB as resistance to at least isoniazid, rifampicin, a fluoroquinolone and one of three injectable second-line drugs (amikacin, kanamycin or capreomycin). First-line drugs in the UK include rifampicin, isoniazid, pyrazinamide and ethambutol.

\section{Data collection}

In addition to data on drug susceptibility collected through the MycobNet collaboration, we retrospectively contacted the reporting clinician to determine the outcome of treatment as well as additional clinical and demographic information on patients with XDRTB. With the exception of one recent XDRTB case, the information was collected more than 3 years after initial diagnosis. Strain typing information based on 15-loci mycobacterial interspersed repetitive units variable number tandem repeats (MIRU-VNTR), performed as described previously, ${ }^{7}$ was collated for all XDRTB cases to investigate the possibility of person to person transmission. MIRU-VNTR typing became available in 1999 and isolates from 
cases diagnosed prior to this date were typed retrospectively where available.

\section{Statistical analysis}

Analysis of second-line drug susceptibility results among MDRTB cases was carried out to examine long-term trends and determine the extent of XDRTB. Proportions were calculated and, where appropriate, an extension of the Wilcoxon rank-sum test for trend across ordered groups was used to assess the statistical significance of trends.

\section{RESULTS}

Between 1995 and 2007, a total of 678 MDRTB cases were reported to the MycobNet collaboration, an average of 52 cases per year. The median age of the group was 32 years (interquartile range 26-43 years) and 59\% (377/639 cases where gender was known) were male. Table 1 shows the proportion of MDRTB cases resistant to second-line antituberculosis drugs. Among MDRTB isolates, levels of resistance to amikacin, capreomycin, ciprofloxacin, cycloserine, ethionamide and PAS were 5.5, 3.4, 5.6, 5.1, 14.0 and $16.7 \%$, respectively. Where tested, resistance to rifabutin and streptomycin was very high. Resistance to the first-line drugs, pyrazinamide and ethambutol was $28.0 \%$ and $36.1 \%$, respectively.

\section{XDRTB cases}

Six XDRTB cases $(0.9 \%$ of MDR cases) were identified during this period. Two further cases of XDRTB were reported in 2008. Of the eight cases, six were male and all were adults (table 2). Three cases were of white ethnic group and six were known to be born outside the UK. Five cases had pulmonary disease with or without extrapulmonary involvement, and six cases were not known or suspected to be HIV positive. All cases had isolates resistant to a variety of second-line drugs with a minimum resistance to five drugs. Five individuals died of tuberculosis within 3 years of diagnosis and three are still on treatment (including the two diagnosed in 2008). Strain typing data were available for five out of the eight XDR isolates; all five were distinguishable using 15-loci MIRU-VNTR. A search of the national strain typing database identified a limited number of

Table 1 Second-line antituberculosis drug resistance among multidrugresistant isolates of $M$ tuberculosis complex, UK, 1995-2007

\begin{tabular}{llr}
\hline Class & Drug & Resistant/tested* (\%) \\
\hline Fluoroquinolones & Ciprofloxacin & $34 / 610(5.6)$ \\
& Moxifloxacin & $1 / 24(4.2)$ \\
& Ofloxacin & $1 / 26(3.9)$ \\
Serine analogues & Any fluoroquinolone & $36 / 647(5.6)$ \\
Aminoglycosides & Cycloserine & $28 / 551(5.1)$ \\
& Amikacin & $32 / 583(5.5)$ \\
Polypeptides & Kanamycin & $1 / 24(4.2)$ \\
Thionamides & Capreomycin & $20 / 592(3.4)$ \\
& Ethionamide & $84 / 601(14.0)$ \\
PAS & Prothionamide & $2 / 5(40.0)$ \\
Others & Any thionamide & $86 / 606(14.2)$ \\
& PAS & $77 / 462(16.7)$ \\
& Clarithromycin & $70 / 587(11.9)$ \\
& Azithromycin & $8 / 92(8.7)$ \\
& Streptomycin & $327 / 636(51.4)$ \\
& Rifabutin & $138 / 160(86.3)$ \\
& Clofazimine & $33 / 94(35.1)$
\end{tabular}

*Where information on testing was available among a total of 678 isolates.

PAS, $p$-aminosalicylic acid.
MDRTB cases which shared indistinguishable MIRU-VNTR type with two of these cases. The 15-digit MIRU-VNTR code for these two isolates was 424352332517333 and 224331432615324, also associated with a further 11 and 3 MDRTB cases, respectively. These MDRTB cases were reported from a geographically widespread area. Stored isolates from three cases could not be recovered. Lack of strain typing data prior to 2003 limits the interpretation of transmission in this context.

A further 12 MDRTB cases had resistance to at least three of the six second-line antituberculosis drugs, fulfilling the initial, but not the subsequently revised, definition for XDRTB. ${ }^{8}$

\section{DISCUSSION}

The level of XDRTB in the UK remains low despite the occurrence of about 50 new MDRTB cases each year and an increase in the incidence of tuberculosis over the last two decades. Eight patients between January 1995 and October 2008 had drug susceptibility results consistent with the current definition of XDRTB. Five of the eight cases died of their disease.

The low levels of XDRTB in the UK compared with other European countries ${ }^{2}$ may reflect minimal incidence of secondand third-line drug resistance in the country of origin of the majority of non-UK-born tuberculosis patients. Alternatively, this may be due to high levels of appropriate clinical management of patients with MDRTB. ${ }^{4}$ Our finding of poor clinical outcome in these patients with XDRTB is consistent with previous studies where a large proportion of cases died of tuberculosis, ${ }^{29}$ especially among individuals co-infected with HIV. In contrast to the South African experience, our patient population had, as far as is known, low levels of HIV infection.

There was no evidence of transmission of XDRTB or from an MDRTB strain with the same MIRU-VNTR pattern, based on typing results. This cannot, however, be excluded as six cases were non-UK born and could have acquired the infection from an MDRTB or XDRTB case outside the UK. Due to the lack of strain typing data prior to 2003, we cannot exclude the possibility that some of the isolates identified prior to widespread typing were responsible for transmission. We were also unable to obtain detailed clinical information on other factors contributing to the death of these patients due to the retrospective nature of this study.

The poor outcome in these patients with XDRTB, however, suggests that UK clinicians should ensure that a package of care which includes directly observed treatment is available for all patients with MDRTB to prevent the development of XDRTB. The experience of most clinicians is likely to be limited, even in parts of the UK with a higher incidence of tuberculosis, given the small number of cases. As the management of these patients is complex, the use of second-line antituberculosis drugs, especially fluoroquinolones, kanamycin, amikacin and capreomycin, in patients infected with MDRTB strains should always be undertaken by those with appropriate experience and expertise. ${ }^{10}$

The management of cases of MDRTB involves the choice of a combination of second-line antituberculosis drugs, and occasionally first-line agents, which should be based on drug susceptibility tests. ${ }^{11}$ It is sometimes necessary to start secondline treatment while awaiting these results. Our data suggest that certain drugs, such as rifabutin and clofazimine, are unlikely to be useful for empirical treatment in the UK in this situation. In addition, $>60 \%$ of MDRTB isolates and three XDRTB cases were susceptible to ethambutol, a drug which has synergistic effects with others due to its action on the cell 
Table 2 Characteristics of extensively drug-resistant tuberculosis cases in the UK, 1995-2008

\begin{tabular}{|c|c|c|c|c|c|c|c|}
\hline Year & Sex & Age & $\begin{array}{l}\text { Ethnicity and } \\
\text { place of birth }\end{array}$ & Previous TB? & $\begin{array}{l}\text { Drug resistance } \\
\text { additional to UK } \\
\text { first-line agents* }\end{array}$ & Site of disease & Outcome \\
\hline 2008 & Female & $20-29$ & White, non-UK & No & $\begin{array}{l}\text { Kan, Mox, Ofl, PAS, } \\
\text { Strep }\end{array}$ & Pulmonary & $\begin{array}{l}\text { Still on } \\
\text { treatment }\end{array}$ \\
\hline 2008 & Male & $20-29$ & $\begin{array}{l}\text { Black African, } \\
\text { non-UK }\end{array}$ & Yes & $\begin{array}{l}\text { AK, Cap, Eth, Kan, Ofl, } \\
\text { Pro, Strep }\end{array}$ & Pulmonary & $\begin{array}{l}\text { Still on } \\
\text { treatment }\end{array}$ \\
\hline 2003 & Male & $30-39$ & White, UK & No & Cap, Cip, Eth & Pulmonary & $\begin{array}{l}\text { Still on } \\
\text { treatment }\end{array}$ \\
\hline 2002 & Male & $30-39$ & White, non-UK & No & $\begin{array}{l}\text { Cap, Cip, Cla, Clo, } \\
\text { Cyc, RB }\end{array}$ & $\begin{array}{l}\text { Cervical Lymph } \\
\text { Node }\end{array}$ & Died of TB \\
\hline 1999 & Male & $20-29$ & $\begin{array}{l}\text { Indian } \\
\text { subcontinent, } \\
\text { non-UK }\end{array}$ & Yes & $\begin{array}{l}\text { AK, Cap, Cip, Cla, Clo, } \\
\text { Eth, RB }\end{array}$ & Pulmonary & Died of TB \\
\hline 1997 & Male & $40-49$ & $\begin{array}{l}\text { Indian } \\
\text { subcontinent, } \\
\text { non-UK }\end{array}$ & Yes & $\begin{array}{l}\text { AK, Cap, Cip, Cla, Clo, } \\
\text { Cyc, PAS, Strep }\end{array}$ & Pulmonary & Died of TB \\
\hline 1997 & Male & 80-89 & Unknown & Unknown & Сap, Cip, Cyc, Strep & Abdominal & Died of TB \\
\hline 1995 & Female & $50-59$ & $\begin{array}{l}\text { Indian } \\
\text { subcontinent, } \\
\text { non-UK }\end{array}$ & Unknown & AK, Cip, Cla, RB, Strep & Pulmonary & Died of TB \\
\hline
\end{tabular}

*First-line drugs in the UK include rifampicin, isoniazid, pyrazinamide and ethambutol.

AK, amikacin; Cap, capreomycin; Cip, ciprofloxacin; Cla, clarithromycin; Clo, clofazimine; Cyc, cycloserine; Eth, ethionamide; Kan, kanamycin; Ofl, ofloxacin; Pro, prothionamide; RB, rifabutin; Strep, streptomycin; TB, tuberculosis.

membrane of mycobacteria, ${ }^{12}$ allowing the use of this agent in such patients. Newer fluoroquinolones such as moxifloxacin should still be considered in the management of these patients as there is evidence that the efficacy of later-generation fluoroquinolones may be preserved, despite resistance to ciprofloxacin. ${ }^{13}$ The Peruvian study suggests that clinicians may also want to reinforce regimens with pyrazinamide and ethambutol with clarithromycin, clofazimine and rifabutin. ${ }^{13}$ Where drug susceptibility information on these agents is available, our data suggest that these agents may have a role in the management of XDRTB.

This study is limited by the small number of cases of XDRTB reported. A further limitation relates to the lack of information on the long-term treatment outcome for all cases of MDRTB to allow a comparison with the patients with XDRTB. National surveillance is being developed to collect information on clinical outcome at 24 months to inform future analysis. As in other developed countries, not all cases of tuberculosis in the UK are culture confirmed. These data therefore represent the susceptibility results from those with a proven bacterial culture only. Nevertheless, this sample included all culture-confirmed cases and is unlikely to be affected by survival bias which many XDRTB studies may be prone to. ${ }^{13}$

Despite the low incidence of MDRTB and XDRTB in the UK, this study found high case fatality among XDRTB patients. A recent postal survey by the British Thoracic Society (BTS) suggested that clinicians with no experience of managing MDRTB remain willing to treat these patients. ${ }^{14}$ Evidence from countries with high rates of drug resistance that have successfully tackled this problem suggests that an alternative approach is likely to be more successful. ${ }^{15}$ The BTS has recently been commissioned by the Department of Health to set up multidisciplinary tuberculosis teams (MDT) working in every area of England and Wales, together with an expert clinical advisory service to underpin and advise the MDTs as needed. In tandem with this, a group of UK clinicians have recently started a national MDRTB service, providing advice on a voluntary basis to colleagues. ${ }^{16}$ The aim is that these developments will help reduce the likelihood that cases of MDRTB will emerge, that they are detected promptly when they do occur, that they are managed effectively (including choice of antituberculosis treatment) and, as a result, their outcomes are improved with minimal occurrence of further second-line antituberculosis drug resistance.

Acknowledgements: We would like to acknowledge all those who provide information on tuberculosis in the UK to the Health Protection Agency and specifically to clinicians and health protection staff who aided the retrospective identification of information on these cases. We are grateful to Louise Bradshaw who maintains the MycobNet database.

IA had full access to all the data in the study and takes responsibility for the integrity of the data and the accuracy of the data analysis. All authors satisfy the minimum requirement for authorship and have approved the final version of the paper.

Competing interests: None.

Ethics approval: The Health Protection Agency has Patient Information Advisory Group approval to hold and analyse national surveillance data for public health purposes under Section 60 of the Health and Social Care Act 2001.

\section{REFERENCES}

1. Park MM, Davis AL, Schluger NW, et al. Outcome of MDR-TB patients, 1983-1993. Prolonged survival with appropriate therapy. Am J Respir Crit Care Med 1996;153:317-24.

2. Migliori GB, Lange C, Centis R, et al. Resistance to second-line injectables and treatment outcomes in multidrug-resistant and extensively drug-resistant tuberculosis cases. Eur Respir J 2008;31:1155-9.

3. Goble M, Iseman MD, Madsen LA, et al. Treatment of 171 patients with pulmonary tuberculosis resistant to isoniazid and rifampin. N Engl J Med 1993;328:527-32.

4. Drobniewski F, Eltringham I, Graham C, et al. A national study of clinical and laboratory factors affecting the survival of patients with multiple drug resistant tuberculosis in the UK. Thorax 2002;57:810-6.

5. The WHO/IUATLD Global Project on Anti-Tuberculosis Drug Resistance Surveillance. Anti-tuberculosis drug resistance in the world. Report No. 4. WHO/ HTM/TB/2008.394. Geneva, 2008.

6. Kruijshaar ME, Watson JM, Drobniewski F, et al. Increasing antituberculosis drug resistance in the United Kingdom: analysis of National Surveillance Data. BMJ 2008;336:1231-4.

7. Frothingham R, Meeker-O'Connell WA. Genetic diversity in the Mycobacterium tuberculosis complex based on variable numbers of tandem DNA repeats. Microbiology 1998;144:1189-96.

8. Wright A, Bai G, Barerra L, et al. Emergence of Mycobacterium tuberculosis with extensive resistance to second-line drugs-worldwide, 2000-2004. MMWR Morb Mortal Wkly Rep 2006;55:301-5.

9. Gandhi NR, Moll A, Sturm AW, et al. Extensively drug-resistant tuberculosis as a cause of death in patients co-infected with tuberculosis and HIV in a rural area of South Africa. Lancet 2006;368:1575-80.

10. Department of Health. Tuberculosis prevention and treatment: a toolkit for planning, commissioning and delivering high-quality services in England. Crown Copyright, 2007 
11. Crofton J, Chaulet P, Maher D. Guidelines for the management of drug resistant tuberculosis. WHO/TB/96.210 (Rev.1). Geneva, 1996.

12. Belanger AE, Besra GS, Ford ME, et al. The embAB genes of Mycobacterium avium encode an arabinosyl transferase involved in cell wall arabinan biosynthesis that is the target for the antimycobacterial drug ethambutol. Proc Natl Acad Sci USA 1996;93:11919-24.

13. Mitnick CD, Shin SS, Seung KJ, et al. Comprehensive treatment of extensively drugresistant tuberculosis. N Engl J Med 2008;359:563-74.
14. British Thoracic Society and the All Party Parliamentary Working Group on TB. Putting tuberculosis on the local agenda. London, 2008.

15. Nathanson E, Lambregts-van Weezenbeek C, Rich ML, et al. Multidrug-resistant tuberculosis management in resource-limited settings. Emerg Infect Dis 2006;12:1389-97.

16. Davies PD, Cullen D. Service for drug resistant tuberculosis exists in the UK. BMJ 2008:336:1324

Pulmonary puzzle

\section{ANSWER}

From the question on page 483

Scar tissue has caused cicatricial supraglottic stenosis from histologically confirmed mucous membrane pemphigoid.

Dysphonia (from anterior glottic scarring) and dyspnoea (from posterior scarring) may progress to severe stenosis, stridor and life-threatening airway obstruction. Laryngeal stenosis predominantly affects children, either as a result of a congenital glottic web or papillomatosis. The subglottis is the most common site for a stricture secondary to an intubation injury. Inflammatory causes include croup, epiglottitis and laryngotracheobronchitis. Adult causes are shown in box $1 .{ }^{1}$

Mucous membrane pemphigoid is a rare chronic systemic autoimmune disease affecting the mucous membranes. Antibodies bind to the basement membrane causing subepidermal bullae which rupture with scarring. There is a preponderance of females and the sixth decade. ${ }^{2}$ Complications include ocular lesions (75\% of patients) with blindness in up to $20 \%,{ }^{3}$ oesophageal strictures, laryngeal stenoses $(8 \%)^{4}$ and bronchial stenoses. Flow-volume loops may show airflow disruption. Ocular, laryngeal, oesophageal, nasopharyngeal and genital involvement are deemed high risk for progression carrying a worse prognosis, ${ }^{3}$ the anti-epigrilin (laminin 5) variant particularly being associated with malignancy.

Trials of management are few and inconclusive, ${ }^{5}$ and relapse and progression are common. Supraglottic stenosis may require repeated laser therapy, adjuvant mitomycin C or, ultimately, tracheostomy. In this case, the stenosis remains under review.

Supraglottic obstruction is illustrated as a rare cause of exertional dyspnoea.

Thorax 2009;64:515. doi:10.1136/thx.2008.101006a

\section{REFERENCES}

1. Krishna PD, Malone JP. Isolated adult supraglottic stenosis: surgical treatment and possible etiologies. Am J Otolaryngol 2006;27:355-7.
Box 1 Laryngeal obstruction or stenosis: aetiology and reported anatomical sites

\section{Supraglottic}

- Carcinomas (95\% squamous)

- Relapsing polychondritis

- Mucous membrane pemphigoid

- Diphtheria

- Epiglottitis

Transglottic

- Carcinomas

- Papillomatosis

- Sarcoidosis

- Tuberculosis

- Perichondritis and radionecrosis

- Fracture

Subglottic

- Carcinomas

- Wegener granulomatosis

- Intubation injury

\section{Other}

- Carcinoid, amyloid, Kaposi sarcoma, metastases, gastrooesophageal reflux.

2. Boedeker CC, Termeer CC, Staats R, et al. Cicatrical pemphigoid in the aerodigestive tract: diagnosis and management in severe laryngeal stenosis. Ann Otol Rhinol Laryngol 2003;112:271-5.

3. Whiteside 0J, Martinez Devesa P, Ali I, et al. Mucous membrane pemphigoid: nasal and laryngeal manifestations. J Laryngol Otol 2003;117:885-8.

4. Gaspar ZS, Wojnarowska F. Cicatrical pemphigoid with severe laryngeal involvement necessitating tracheostomy (laryngeal cicatrical pemphigoid). Clin Exp Dermatol 1996;21:209-10.

5. Sacher C, Hunzelmann N. Cicatrical pemphigoid (mucous membrane pemphigoid) current and emerging therapeutic approaches. Am J Clin Dermatol 2005;6:93-103. 